\section{Kinase race in PD}

\section{By Lev Osherovich, Senior Writer}

It's not every day that academic drug discovery outpaces a company, but a team at The Johns Hopkins University thinks it has done just that by providing the first in vivo proof of concept that pharmacological inhibition of leucine-rich repeat kinase 2 is neuroprotective in a mouse model of Parkinson's disease. The molecules are being optioned by an undisclosed company, which would find itself in a three-horse race with two biotechs that have preclinical programs targeting the kinase in PD: Zenobia Therapeutics Inc. and TauTaTis Inc.

Leucine-rich repeat kinase 2 (LRRK2) became a suspect in PD in 2005 when independent teams identified mutations in the protein in patients with a dominantly inherited form of the disease. Subsequent work showed that about $5 \%$ of PD patients harbor mutations that increase the enzyme's activity, but a lack of good animal models made it hard to test whether inactivating LRRK2 would actually have a disease-modifying effect.

The Johns Hopkins team was able to create such a test. The researchers created a mouse model in which overexpression of mutated Lrrk2 induced degeneration of dopaminergic neurons-a hallmark of PD-and showed that neuron loss can be blocked by a range of small molecule kinase inhibitors.

The results were reported in Nature Medicine. ${ }^{1}$

"Within the paper is the first in vivo model for LRRK2-induced loss of dopamine neurons," said Ted Dawson, team leader and professor of neurodegenerative disease at The Johns Hopkins University Institute for Cell Engineering. "The principal novelty is the proof of concept that inhibitors of LRRK2 are protective against loss of dopamine neurons."

Dawson's team began by screening 84 kinase and phosphatase inhibitors for in vitro activity against LRRK2. The group found eight compounds that decreased LRRK2 autophosphorylation compared with no treatment.

One of the compounds was an inhibitor of CRAF (RAF1), a kinase that is closely related to LRRK2. Dawson's team proceeded to test a panel of CRAF inhibitors that had originally been developed as cancer therapeutics. They found that CRAF inhibitors including Nexavar sorafenib inhibited LRRK2's ability to phosphorylate itself and several model substrates in vitro compared with no treatment.

Onyx Pharmaceuticals Inc. and Bayer AG market Nexavar, an inhibitor of VEGF, CRAF and multiple receptor tyrosine kinases, to treat liver and renal cancer.

The team also found that indirubin-3'-monooxime, an inhibitor of glycogen synthase kinase $3 \beta$ (GSK3B), could inhibit LRRK2.

Cultured mouse neurons pretreated with CRAF inhibitors and indirubin-3'-monooxime had higher tolerance for subsequent expression of a mutant LRRK2 transgene than vector-transfected control neurons. Nexavar and another CRAF inhibitor, a research reagent from GlaxoSmithKline plc called GW-5074, protected cultured neurons from LRRK2 overexpression more effectively than other CRAF inhibitors that had lower in vitro activity.

Finally, the team devised a mouse model of LRRK2 toxicity in which the PD-linked mutant version of the protein was introduced via viral vector into the substantia nigra, the part of the brain most affected by PD. Peripheral injection of GW-5074 and indirubin-3'-monooxime decreased dopaminergic neuron loss caused by the transgene compared with injection of vehicle.

"As far I know, this is the first example where inhibitors of LRRK2 were shown to be neuroprotective," said Vicki Nienaber, president, CSO and founder of Zenobia.

\section{Hit to lead}

The compounds used by Dawson's group could be a good starting point for making better LRRK2 inhibitors. However, other researchers think the effects described in Nature Medicine may stem from the originally intended targets of many of the molecules, such as CRAF and GSK3B.

For example, Santosh D’Mello, professor of molecular and cell biology at The University of Texas at Dallas, said his own studies with GW-5074 suggest that CRAF, rather than LRRK2, may be the principal target for the compound in the brain. D'Mello's team has tested GW-5074 in mouse models of Huntington's disease (HD). ${ }^{2}$

The group's studies suggest that GW-5074 doesn't directly inhibit LRRK2, though his team's assays are different than Dawson's.

"It is likely that neuroprotection by GW-5074 is mediated through another mechanism related to CRAF inhibition, or that inhibition of LRRK2 in vivo is a consequence of reduced CRAF activity," D'Mello wrote in an e-mail to $S c i B X$.

Meanwhile, GSK3B is a PD target in its own right. Neurim Pharmaceuticals Ltd.'s Neu-120, a dual GSK3B and monoamine oxidase B (MAO-B) inhibitor, is in Phase II testing for PD.

Daniel Watterson, a professor of molecular pharmacology and biological chemistry at Northwestern University, said that because many of the compounds described in Nature Medicine inhibit a variety of other kinases, it remains unclear whether their in vivo benefits are really due to LRRK2 inhibition.

Nevertheless, Watterson did say several of the compounds used by the Johns Hopkins team, including indirubin-3'-monooxime and GW-5074, were good starting points for hit-to-lead work because they had high affinity for LRRK2.

"For kinase inhibitors, if you're below $1 \mathrm{mMIC}_{50}$, it's worth investigating," he said. For these molecules to advance to become drug candidates, the $\mathrm{IC}_{50}$ should be optimized to "around $20 \mathrm{nM}$," he said.

\section{Model muddle}

Although the compounds from Dawson's group inhibit LRRK2 in vitro, interpreting the in vivo data becomes complicated because of the nature of his team's PD model. 


\section{ANALYSIS}

Hanno Roder, chairman and president of TauTaTis, said the in vivo model is biased toward showing a therapeutic effect for kinase inhibitors, but this may not recapitulate what goes on in PD patients.

"In this model, the investigators use relatively short-term expression of LRRK2. Within three weeks, neurons die and this can be prevented with these compounds," said Roder. The problem, he said, is that "they've loaded up the cells with LRRK2 until they can't take any more of it, so the experiment is biased to demonstrate that kinase inhibition will fix this."

Dawson said overexpression of mutant LRRK2 captures at least one key feature of PD that is missing in other rodent models-degeneration of dopaminergic neurons.

Roder also said it remains unclear whether inhibiting normal levels of mutant or wild-type LRRK2 alone would have a disease-modifying effect.

TauTaTis' TTT-3002 is an LRRK2 inhibitor in preclinical development for PD. According to Roder, TTT-3002 has nanomolar affinity for LRRK2 and also inhibits other kinase pathways that have been implicated in PD.

\section{Selectivity, permeability, flexibility}

Uncovering the mechanism of action for the compounds used by the Johns Hopkins team may be a moot point if inhibiting kinases in general has a beneficial effect in PD.

Indeed, both Roder and Watterson said it may in fact be desirable to hit multiple kinases, since a variety of signaling pathways are perturbed in the disease.

Roder thinks the biggest obstacle for Dawson and others trying to target LRRK2 will be to strike a balance between selectivity, low toxicity and blood brain barrier (BBB) permeability.

"Finding a compromise between [BBB] permissiveness, tolerability and activity in the multiple pathways involved in pathology has to be done empirically," said Roder.

Watterson recommended testing the compounds described in Nature Medicine in at least one other in vivo disease model.

"You want more than one disease model with a different mechanism of injury," he said. "No one model fully recapitulates disease."

According to Dawson, the work has been patented and optioned to an undisclosed company.

Osherovich, L. SciBX 3(34); doi:10.1038/scibx.2010.1029

Published online Sept. 2, 2010

\section{REFERENCES}

1. Lee, B.D. et al. Nat. Med.; published online Aug. 22, 2010; doi:10.1038/nm.2199

Contact: Ted M. Dawson, The Johns Hopkins University School of Medicine, Baltimore, Md. e-mail: tdawson@jhmi.edu

2. Chen, H.-M. et al. J. Neurochem. 105, 1300-1312 (2008)

\section{COMPANIES AND INSTITUTIONS MENTIONED}

Bayer AG (Xetra:BAY), Leverkusen, Germany

GlaxoSmithKline plc (LSE:GSK; NYSE:GSK), London, U.K.

The Johns Hopkins University, Baltimore, Md.

Neurim Pharmaceuticals Ltd., Tel Aviv, Israel

Northwestern University, Evanston, III.

Onyx Pharmaceuticals Inc. (NASDAQ:ONXX), Emeryville, Calif.

TauTaTis Inc., Jacksonville, Fla.

The University of Texas at Dallas, Dallas, Texas

Zenobia Therapeutics Inc., La Jolla, Calif. 